\title{
Applications of a q-Salagean type operator on multivalent functions
}

\author{
Saqib Hussain ${ }^{1 *}$, Shahid Khan², Muhammad Asad Zaighum² and Maslina Darus ${ }^{3}$
}

\section{"Correspondence:}

saqib_math@yahoo.com

'Department of Mathematics, COMSATS University Islamabad,

Abbottabad Campus, Pakistan Full list of author information is

available at the end of the article

\begin{abstract}
In this paper, we introduce a new class $k-\mathcal{U S}(q, \gamma, m, p), \gamma \in \mathbb{C} \backslash\{0\}$, of multivalent functions using a newly defined $q$-analogue of a Salagean type differential operator. We investigate the coefficient problem, Fekete-Szego inequality, and some other properties related to subordination. Relevant connections of the results presented here with those obtained in the earlier work are also pointed out.
\end{abstract}

MSC: Primary 30C45; secondary 30C50

Keywords: Multivalent functions; 9 derivative operator; Convolution; Conic domain

\section{Introduction}

For a positive integer $p$, let $\mathcal{A}_{p}$ denote the set of all functions $f(z)$ which are analytic and $p$-valent in the open unit disk $E=\{z \in \mathbb{C}:|z|<1\}$ and have series expansion of the form

$$
f(z)=z^{p}+\sum_{n=p+1}^{\infty} a_{n} z^{n} .
$$

Also, let $f * g$ denote the convolution (or Hadamard product) of $f, g \in \mathcal{A}_{p}$ defined as follows:

$$
(f * g)(z)=z^{p}+\sum_{n=p+1}^{\infty} a_{n} b_{n} z^{n},
$$

where $f(z)$ is given by (1.1) and $g(z)=z^{p}+\sum_{n=p+1}^{\infty} b_{n} z^{n}$.

Quite recently, $q$-analysis has influenced the researchers a lot due to rapid applications in mathematics and related fields. In the last century many well-known researchers (for details, see $[1,4,6-10,13,14,21,22,32]$ ) did great work on $q$-calculus and found numerous applications. It is worth mentioning that convolution theory helps many researchers to investigate a number of properties of analytic univalent and multivalent functions. Several differential and integral operators were defined using ordinary derivative; for details, see [29].

Due to growing applications of $q$-calculus, investigators are interested in studying properties of functions using $q$-operators instead of ordinary differential operators; for comprehensive study, we refer to Kanas and Reducanu [15], Mahmood and Darus [19], and

(c) The Author(s) 2018. This article is distributed under the terms of the Creative Commons Attribution 4.0 International License (http://creativecommons.org/licenses/by/4.0/), which permits unrestricted use, distribution, and reproduction in any medium, provided you give appropriate credit to the original author(s) and the source, provide a link to the Creative Commons license, and indicate if changes were made. 
Mahmood and Sokol [20]. In this paper we define a $q$-analogue of a Salagean type operator and study its effect on multivalent functions in conic domains.

For any non-negative integer $n$, the $q$-integer number $n$ denoted by $[n]_{q}$ is defined by

$$
[n]_{q}=\frac{1-q^{n}}{1-q}, \quad[0]_{q}=0
$$

For a non-negative integer $n$, the $q$-number shift factorial is defined as

$$
[n]_{q} !=[1]_{q}[2]_{q}[3]_{q} \ldots[n]_{q} \quad\left([0]_{q} !=1\right) .
$$

We note that when $q \rightarrow 1,[n]_{q}$ ! reduces to the classical definition of factorial. In general, $[t]_{q}$ is defined as follows:

$$
[t]_{q}=\frac{1-q^{t}}{1-q}, \quad[0]_{q}=0, \quad q \in(0,1)
$$

For $f \in A$, in [5], the $q$-derivative operator or $q$-difference operator is defined as follows:

$$
\partial_{q} f(z)=\frac{f(q z)-f(z)}{z(q-1)} .
$$

It can easily be seen that

$$
\partial_{q} z^{n}=[n]_{q} z^{n-1}, \quad \partial_{q}\left\{\sum_{n=1}^{\infty} a_{n} z^{n}\right\}=\sum_{n=1}^{\infty}[n]_{q} a_{n} z^{n-1} .
$$

Taking motivation from the above mentioned work, we define new convolution operators as follows.

Let

$$
\Phi(p, q, m, z)=z^{p}+\sum_{n=p+1}^{\infty}[n+(p-1)]_{q}^{m} z^{n} .
$$

Using the functions $\Phi(p, q, m, z)$ and the definition of $q$-derivative along with the idea of convolution, we now define the following differential operator $\mathcal{S}_{q, p}^{m} f(z): \mathcal{A}_{p} \rightarrow \mathcal{A}_{p}$ for multivalent functions

$$
\begin{aligned}
\mathcal{S}_{q, p}^{m} f(z) & =\Phi(p, q, m, z) * f(z), \quad m \in N \cup\{0\}, \\
& =z^{p}+\sum_{n=p+1}^{\infty}[n+(p-1)]_{q}^{m} a_{n} z^{n}, \\
& =z^{p}+\sum_{n=p+1}^{\infty} \psi_{n} a_{n} z^{n},
\end{aligned}
$$

where

$$
\psi_{n}=[n+(p-1)]_{q}^{m} .
$$


For $p=1$, the operator $\mathcal{S}_{q, p}^{m} f(z)$ reduces to the Salagean $q$-differential operator defined by Govindaraj and Sivasubramanian [11], and for $p=1, q \rightarrow 1$, the operator $\mathcal{S}_{q, p}^{m} f(z)$ reduces to the Salagean differential operator defined by Salagean [26].

Taking motivation from [12] and using (1.3), we define a new class $k-\mathcal{U S}(q, \gamma, m, p)$ of multivalent functions as follows.

Throughout paper we shall assume $k \geq 0, m \in N \cup\{0\}, q \in(0,1), \gamma \in \mathbb{C} \backslash\{0\}$, and $p \in N$.

Definition 1.1 A function $f(z) \in \mathcal{A}_{p}$ is in the class $k-\mathcal{U S}(q, \gamma, m, p)$ if it satisfies the condition

$$
\operatorname{Re}\left\{1+\frac{1}{\gamma}\left\{\frac{1}{[p]_{q}}\left(\frac{z \partial_{q} S_{q, p}^{m} f(z)}{S_{q, p}^{m} f(z)}\right)-1\right\}\right\}>k\left|\frac{1}{\gamma}\left\{\frac{1}{[p]_{q}}\left(\frac{z \partial_{q} S_{q, p}^{m} f(z)}{S_{q, p}^{m} f(z)}\right)-1\right\}\right|, \quad z \in E .
$$

By taking specific values of parameters, we obtain many important subclasses studied by various authors in earlier papers. Here we enlist some of them.

(i) For $p=1$, the class $k-\mathcal{U S}(q, \gamma, m, p)$ reduces to the class $k-\mathcal{U S}(q, \gamma, m)$ studied by Saqib et al. [12].

(ii) For $p=1, m=0, k=0$, and $\gamma \in \mathbb{C} \backslash\{0\}$, the class $k-\mathcal{U S}(q, \gamma, m, p)$ reduces to the class $\mathcal{S}_{q}^{*}(\gamma)$ studied by Seoudy and Aouf [27].

(iii) For $p=1, m=0, k=0$, and $\gamma=\frac{1}{1-\alpha}$, with $0 \leq \alpha<1$, the class $k-\mathcal{U S}(q, \gamma, m, p)$ reduces to the class $\mathcal{S}_{q}^{*}(\alpha)$ studied by Agrawal and Sahoo [2].

(iv) For $p=1, m=0, q \rightarrow 1$, and $\gamma=\frac{1}{1-\alpha}$, with $0 \leq \alpha<1$, the class $k-\mathcal{U S}(q, \gamma, m, p)$ reduces to the class $\mathcal{S D}(k, \alpha)$ studied by Shams et al. [28].

(v) For $p=1, m=0, q \rightarrow 1$, and $\gamma=\frac{2}{1-\alpha}$, with $0 \leq \alpha<1$, the $\operatorname{class} k-\mathcal{U S}(q, \gamma, m, p)$ reduces to the class $\mathcal{K D}(k, \alpha)$ studied by Owa et al. [24].

(vi) For $p=1, k=1, m=0, q \rightarrow 1$, and $\gamma=\frac{1}{1-\alpha}$, with $0 \leq \alpha<1$, the class $k-\mathcal{U S}(q, \gamma, m, p)$ reduces to the class $\mathcal{S}(\alpha)$ studied by Ali et al. [3].

(vii) For $p=1, k=1, m=0, q \rightarrow 1$, and $\gamma=\frac{2}{1-\alpha}$, with $0 \leq \alpha<1$, the class $k-\mathcal{U S}(q, \gamma, m, p)$ reduces to the class $\mathcal{K}(\alpha)$ studied by Ali et al. [3].

(viii) For $p=1, m=0, q \rightarrow 1$, the class $k-\mathcal{U S}(q, \gamma, m, p)$ reduces to the class $\mathcal{K}-\mathcal{S} \mathcal{T}$ introduced by Kanas and Wisniowska [17].

(ix) For $p=1, k=0, m=0, q \rightarrow 1$, and $\gamma=\frac{1}{1-\alpha}$, with $0 \leq \alpha<1$, the class $k-\mathcal{U S}(q, \gamma, m, p)$ reduces to the class $\mathcal{S}^{*}(\alpha)$, a well-known class of starlike functions of order $\alpha$, respectively.

Geometric interpretation. A function $f(z) \in \mathcal{A}_{p}$ is in the class $k-\mathcal{U S}(q, \gamma, m, p)$ if and only if $\frac{1}{[p]_{q}}\left(\frac{z \partial_{q} S_{q, f}^{m} f(z)}{S_{q, p}^{m} f(z)}\right)$ takes all the values in the conic domain $\Omega_{k, \gamma}=h_{k, \gamma}(E)$ such that

$$
\Omega_{k, \gamma}=\gamma \Omega_{k}+(1-\alpha)
$$

where

$$
\Omega_{k}=\left\{u+i v: u>k \sqrt{(u-1)^{2}+v^{2}}\right\} .
$$

Since $h_{k, \gamma}(z)$ is convex univalent, so the above definition can be written as

$$
\frac{1}{[p]_{q}}\left(\frac{z \partial_{q} S_{q, p}^{m} f(z)}{S_{q, p}^{m} f(z)}\right) \prec h_{k, \gamma}(z)
$$


where

$$
h_{k, \gamma}(z)= \begin{cases}\frac{1+z}{1-z}, & \text { for } k=0, \\ 1+\frac{2 \gamma}{\pi^{2}}\left(\log \frac{1+\sqrt{z}}{1-\sqrt{z}}\right)^{2}, & \text { for } k=1, \\ 1+\frac{2 \gamma}{1-k^{2}} \sinh ^{2}\left\{\left(\frac{2}{\pi} \arccos k\right) \arctan h \sqrt{z}\right\}, & \text { for } 0<k<1, \\ 1+\frac{\gamma}{k^{2}-1} \sin \left(\frac{\pi}{2 R(t)} \int_{0}^{\frac{u(z)}{\sqrt{t}}} \frac{1}{\sqrt{1-x^{2}} \sqrt{1-(t x)^{2}}} d x\right)+\frac{\gamma}{1-k^{2}}, & \text { for } k>1 .\end{cases}
$$

The boundary $\partial \Omega_{k, \gamma}$ of the above set becomes an imaginary axis when $k=0$, and a hyperbola when $0<k<1$. For $k=1$, the boundary $\partial \Omega_{k, \gamma}$ becomes a parabola and it is an ellipse when $k>1$ and in this case where

$$
u(z)=\frac{z-\sqrt{t}}{1-\sqrt{t} z}, \quad z \in E,
$$

and $t \in(0,1)$ is chosen such that $k=\cosh \left(\pi K^{\prime}(t) /(4 K(t))\right)$. Here $K(t)$ is Legendre's complete elliptic integral of the first kind and $K^{\prime}(t)=K\left(\sqrt{1-t^{2}}\right)$, and $K^{\prime}(t)$ is the complementary integral of $K(t)$ (for details, see $[16,17,23]$ ). Moreover, $h_{k, \gamma}(E)$ is convex univalent in $E$, see $[16,17]$. All of these curves have the vertex at the point $\frac{k+\gamma}{k+1}$.

\section{A set of lemmas}

Each of the following lemmas will be needed in our present investigation.

Lemma 2.1 ([25]) Let $h(z)=\sum_{n=1}^{\infty} h_{n} z^{n} \prec F(z)=\sum_{n=1}^{\infty} d_{n} z^{n}$ in E. If $F(z)$ is convex univalent in $E$, then

$$
\left|h_{n}\right| \leq\left|d_{1}\right|, \quad n \geq 1 .
$$

Lemma $2.2([31])$ Let $k \in[0, \infty)$ and let $h_{k, \gamma}$ be defined (1.6). If

$$
\begin{aligned}
& h_{k, \gamma}(z)=1+Q_{1} z+Q_{2} z^{2}+\cdots, \\
& Q_{1}= \begin{cases}\frac{2 \gamma A^{2}}{1-k^{2}}, & 0 \leq k<1, \\
\frac{8 \gamma}{\pi^{2}}, & k=1, \\
\frac{\pi^{2} \gamma}{4(1+t) \sqrt{t} K^{2}(t)\left(k^{2}-1\right)}, & k>1,\end{cases} \\
& Q_{2}= \begin{cases}\frac{A^{2}+2}{3} Q_{1}, & 0 \leq k<1, \\
\frac{2}{3} Q_{1}, & k=1, \\
\frac{4 K^{2}(t)\left(t^{2}+6 t+1\right)-\pi^{2}}{24 K^{2}(t)(1+t) \sqrt{t}} Q_{1}, & k>1,\end{cases}
\end{aligned}
$$

where $A=\frac{2 \cos ^{-1} k}{\pi}$, and $t \in(0,1)$ is chosen such that $k=\cosh \left(\frac{\pi K^{\prime}(t)}{K(t)}\right), K(t)$ is Legendre's complete elliptic integral of the first kind.

Lemma 2.3 ([18]) Let $h(z)=1+\sum_{n=1}^{\infty} c_{n} z^{n}$ be analytic in E and satisfy $\operatorname{Re}\{h(z)\}>0$ for $z$ in $E$. Then the following sharp estimate holds:

$$
\left|c_{2}-\mu c_{1}^{2}\right| \leq 2 \max \{1,|2 \mu-1|\}, \quad \forall \mu \in \mathbb{C} .
$$




\section{Main results}

In this section, we will prove our main results.

Theorem 3.1 Let $f(z) \in k-\mathcal{U S}(q, \gamma, m, p)$. Then

$$
S_{q, p}^{m} f(z) \prec z \exp \int_{0}^{z} \frac{p\left\{h_{k, \gamma}(w(z))\right\}-1}{\zeta} d \xi,
$$

where $w(z)$ is analytic in $E$ with $w(0)=0$ and $|w(z)|<1$. Moreover, for $|z|=\rho$, we have

$$
\exp \left(\int_{0}^{1} \frac{p\left\{h_{k, \gamma}(-\rho)\right\}-1}{\rho} d \rho\right) \leq\left|\frac{S_{q, p}^{m} f(z)}{z}\right| \leq \exp \left(\int_{0}^{1} \frac{p\left\{h_{k, \gamma}(\rho)\right\}-1}{\rho} d \rho\right)
$$

where $h_{k, \gamma}(z)$ is defined by (1.6).

Proof If $f(z) \in k-\mathcal{U S}(q, \gamma, m, p)$, then using identity (1.5), we obtain

$$
\begin{aligned}
& \frac{1}{p}\left(\frac{z \partial_{q} S_{q, p}^{m} f(z)}{S_{q, p}^{m} f(z)}\right) \prec h_{k, \gamma}(z), \\
& \frac{1}{p}\left(\frac{z \partial_{q} S_{q, p}^{m} f(z)}{S_{q, p}^{m} f(z)}\right)=h_{k, \gamma}(w(z)), \\
& \frac{\partial_{q} S_{q, p}^{m} f(z)}{S_{q, p}^{m} f(z)}-\frac{1}{z}=\frac{p\left\{h_{k, \gamma}(w(z))\right\}-1}{z} .
\end{aligned}
$$

For some function $w(z)$ is analytic in $E$ with $w(0)=0$ and $|w(z)|<1$. Integrating (3.3) and after some simplification, we have

$$
S_{q, p}^{m} f(z) \prec z \exp \int_{0}^{z} \frac{p\left\{h_{k, \gamma}(w(z))\right\}-1}{\zeta} d \xi
$$

This proves (3.1). Noting that the univalent function $h_{k, \gamma}(z)$ maps the disk $|z|<\rho(0<\rho \leq$ 1 ) onto a region which is convex and symmetric with respect to the real axis, we see

$$
h_{k, \gamma}(-\rho|z|) \leq \operatorname{Re}\left\{h_{k, \gamma}(w(\rho z)\} \leq h_{k, \gamma}(\rho|z|) \quad(0<\rho \leq 1, z \in E) .\right.
$$

Using (3.4) and (3.5) gives

$$
\begin{aligned}
\int_{0}^{1} \frac{p\left\{h_{k, \gamma}(-\rho|z|)\right\}-1}{\rho} d \rho & \leq \operatorname{Re} \int_{0}^{1} \frac{p\left\{h_{k, \gamma}(w(\rho(z))\}-1\right.}{\rho} d \rho \\
& \leq \int_{0}^{1} \frac{p\left\{h_{k, \gamma}(\rho|z|)\right\}-1}{\rho} d \rho
\end{aligned}
$$

for $z \in E$. Consequently, subordination (3.4) leads to

$$
\begin{aligned}
& \int_{0}^{1} \frac{p\left\{h_{k, \gamma}(-\rho|z|)\right\}-1}{\rho} d \rho \leq \log \left|\frac{S_{q, p}^{m} f(z)}{z}\right| \leq \int_{0}^{1} \frac{p\left\{h_{k, \gamma}(\rho|z|)\right\}-1}{\rho} d \rho, \\
& h_{k, \gamma}(-\rho) \leq h_{k, \gamma}(-\rho|z|), \quad h_{k, \gamma}(\rho|z|) \leq h_{k, \gamma}(\rho)
\end{aligned}
$$


implies that

$$
\begin{aligned}
\exp \int_{0}^{1} \frac{p\left\{h_{k, \gamma}(-\rho)\right\}-1}{\rho} d \rho & \leq\left|\frac{S_{q, p}^{m} f(z)}{z}\right| \\
& \leq \exp \int_{0}^{1} \frac{p\left\{h_{k, \gamma}(\rho)\right\}-1}{\rho} d \rho .
\end{aligned}
$$

This completes the proof.

When $p=1$, we have the following known result proved by Saqib et al. in [12].

Corollary 3.2 Let $f(z) \in k-\mathcal{U S}(q, \gamma, m)$. Then

$$
S_{q}^{m} f(z) \prec z \exp \int_{0}^{z} \frac{h_{k, \gamma}(w(\xi))-1}{\zeta} d \xi
$$

where $w(z)$ is analytic in $E$ with $w(0)=0$ and $|w(z)|<1$. Moreover, for $|z|=\rho$, we have

$$
\begin{aligned}
\exp \left(\int_{0}^{1} \frac{h_{k, \gamma}(-\rho)-1}{\rho} d \rho\right) & \leq\left|\frac{S_{q}^{m} f(z)}{z}\right| \\
& \leq \exp \left(\int_{0}^{1} \frac{h_{k, \gamma}(\rho)-1}{\rho} d \rho\right)
\end{aligned}
$$

where $h_{k, \gamma}(z)$ is defined by (1.6).

Theorem 3.3 Iff $(z) \in k-\mathcal{U S}(q, \gamma, m, p)$, then

$$
\left|a_{p+1}\right| \leq \frac{\delta}{\left\{[p+1]_{q}-p\right\} \psi_{p+1}}
$$

and

$$
\left|a_{n+p-1}\right| \leq \frac{\delta}{\left\{[n+p-1]_{q}-p\right\} \psi_{n+p-1}} \prod_{j=1}^{n-2}\left(1+\frac{\delta}{\left\{[j+p]_{q}-p\right\}}\right) \text { for } n=3,4, \ldots
$$

where $\delta=p\left|Q_{1}\right|$ with $Q_{1}$ given by (2.2).

Proof Let

$$
\begin{aligned}
& \frac{1}{[p]_{q}}\left(\frac{z \partial_{q} S_{q, p}^{m} f(z)}{S_{q, p}^{m} f(z)}\right)=h(z), \\
& z \partial_{q} S_{q, p}^{m} f(z)=[p]_{q} S_{q, p}^{m} f(z) h(z),
\end{aligned}
$$

where $h(z)$ is analytic in $E$ and $h(0)=1$. Let $h(z)=1+\sum_{n=1}^{\infty} c_{n} z^{n}$ and $S_{q, p}^{m} f(z)$ be given by (1.3). Then (3.8) becomes

$$
z^{p}+\sum_{n=p+1}^{\infty}[n]_{q} \psi_{n} a_{n} z^{n}=p\left(\sum_{n=0}^{\infty} c_{n} z^{n}\right)\left(z^{p}+\sum_{n=p+1}^{\infty} \psi_{n} a_{n} z^{n}\right) .
$$


Now comparing the coefficients of $z^{n+p-1}$, we obtain

$$
\begin{aligned}
& {[n+p-1]_{q} \psi_{n+p-1} a_{n+p-1}=p \psi_{n+p-1} a_{n+p-1}+p\left\{c_{1} \psi_{n+p-2} a_{n+p-2}+\cdots+c_{n-1}\right\},} \\
& \left\{[n+p-1]_{q}-p\right\} \psi_{n+p-1} a_{n+p-1}=p\left\{c_{1} \psi_{n+p-2} a_{n+p-2}+\cdots+c_{n-1}\right\} .
\end{aligned}
$$

Taking the absolute on both sides and then applying the coefficient estimates $\left|c_{n}\right| \leq\left|Q_{1}\right|$, see in [23], we have

$$
\left|a_{n+p-1}\right| \leq \frac{p\left|Q_{1}\right|}{\left\{[n+p-1]_{q}-p\right\} \psi_{n+p-1}}\left\{1+\psi_{p+1}\left|a_{p+1}\right|+\cdots+\psi_{n+p-2}\left|a_{n+p-2}\right|\right\} .
$$

Let us take $\delta=p\left|Q_{1}\right|$, then we have

$$
\left|a_{n+p-1}\right| \leq \frac{\delta}{\left\{[n+p-1]_{q}-p\right\} \psi_{n+p-1}}\left\{1+\psi_{p+1}\left|a_{p+1}\right|+\cdots+\psi_{n+p-2}\left|a_{n+p-2}\right|\right\} .
$$

We apply mathematical induction on (3.9), so for $n=2$ in (3.9), we have

$$
\left|a_{p+1}\right| \leq \frac{\delta}{\left\{[p+1]_{q}-p\right\} \psi_{p+1}}
$$

which shows that (3.7) holds for $n=2$. Now consider the case $n=3$ in (3.9), we have

$$
\left|a_{p+2}\right| \leq \frac{\delta}{\left\{[p+2]_{q}-p\right\} \psi_{p+2}}\left\{1+\psi_{p+1}\left|a_{p+1}\right|\right\} .
$$

Using (3.10), we have

$$
\left|a_{p+2}\right| \leq \frac{\delta}{\left\{[p+2]_{q}-p\right\} \psi_{p+2}}\left\{1+\frac{\delta}{[p+1]_{q}-p}\right\}
$$

which shows that (3.7) holds for $n=3$. Let us assume that (3.7) is true for $n \leq t$, that is,

$$
\left|a_{t+p-1}\right| \leq \frac{\delta}{\left\{[t+p-1]_{q}-p\right\} \psi_{t+p-1}} \prod_{j=1}^{t-2}\left(1+\frac{\delta}{[j+p]_{q}-p}\right) \quad \text { for } n=3,4, \ldots
$$

Consider

$$
\begin{aligned}
\left|a_{t+p}\right| & \leq \frac{\delta}{\left\{[t+p]_{q}-p\right\} \psi_{t+p}}\left\{1+\psi_{p+1}\left|a_{p+1}\right|+\cdots \psi_{t+p-1}\left|a_{t+p-1}\right|\right\} \\
& \leq \frac{\delta}{\left\{[t+p]_{q}-p\right\} \psi_{t+p}}\left\{\begin{array}{c}
1+\frac{\delta}{[p+1]_{q}-p}+\frac{\delta}{[p+2]_{q}-p}\left(1+\frac{\delta}{[p+1]_{q}-p}\right)+\cdots \\
+\frac{\delta}{\left\{[t+p-1]_{q}-p\right\}} \prod_{j=1}^{t-2}\left(1+\frac{\delta}{[j+p]_{q}-p}\right)
\end{array}\right\} \\
& =\frac{\delta}{\left\{[t+p]_{q}-p\right\} \psi_{t+p}} \prod_{j=1}^{t-1}\left(1+\frac{\delta}{[j+p]_{q}-p}\right)
\end{aligned}
$$

which proves the assertion of theorem $n=t+1$. Hence (3.7) holds for all $n, n \geq 3$.

This completes the proof. 
When $p=1$, we have the following known result proved by Saqib et al. in [12].

Corollary 3.4 ([12]) Iff $(z) \in k-\mathcal{U S}(q, \gamma, m)$, then

$$
\left|a_{2}\right| \leq \frac{\delta}{\left\{[2]_{q}-1\right\}[2]_{q}^{m}}
$$

and

$$
\left|a_{n}\right| \leq \frac{\delta}{\left\{[n]_{q}-1\right\}[n]_{q}^{m}} \prod_{j=1}^{n-2}\left(1+\frac{\delta}{[j+1]_{q}-1}\right) \quad \text { for } n=3,4, \ldots
$$

where $\delta=\left|Q_{1}\right|$ with $Q_{1}$ given by (2.2).

Theorem 3.5 Let $0 \leq k<\infty$ be fixed and let $f(z) \in k-\mathcal{U S}(q, \gamma, m, p)$ with the form (1.1). Then, for a complex number $\mu$,

$$
\left|a_{p+2}-\mu a_{p+1}^{2}\right| \leq \frac{p Q_{1}}{2[2 p+1]_{q}^{m}\left\{[p+2]_{q}-p\right\}} \max [1,|2 v-1|],
$$

where

$$
\nu=\frac{1}{2}\left\{1-\frac{Q_{2}}{Q_{1}}-Q_{1}\left(\frac{4 p}{\left\{[p+1]_{q}-p\right\}}-\mu \frac{4 p[2 p+1]_{q}^{m}\left\{[p+2]_{q}-p\right\}}{\left([2 p]_{q}^{m}\right)^{2}\left\{[p+1]_{q}-p\right\}}\right)\right\},
$$

and $\delta=p\left|Q_{1}\right|$, with $Q_{1}$ and $Q_{2}$ given by (2.2) and (2.3).

Proof Let $f(z) \in k-\mathcal{U S}(q, \gamma, m, p)$, then there exists a Schwarz function $w(z)$, with $w(0)=0$ and $|w(z)|<1$, such that

$$
\begin{aligned}
& \frac{1}{[p]_{q}}\left(\frac{z \partial_{q} S_{q, p}^{m} f(z)}{S_{q, p}^{m} f(z)}\right) \prec h_{k, \gamma}(z), \quad z \in E, \\
& \frac{1}{[p]_{q}}\left(\frac{z \partial_{q} S_{q, p}^{m} f(z)}{S_{q, p}^{m} f(z)}\right)=h_{k, \gamma}(w(z)) .
\end{aligned}
$$

Let $h(z) \in \mathcal{P}$ be a function defined as

$$
h(z)=\frac{1+w(z)}{1-w(z)},
$$

which gives

$$
w(z)=\frac{c_{1}}{2} z+\frac{1}{2}\left(c_{2}-\frac{c_{1}^{2}}{2}\right) z^{2}+\cdots
$$

and

$$
h_{k, \gamma}(w(z))=1+\frac{Q_{1} c_{1}}{2} z+\left\{\frac{Q_{2} c_{1}^{2}}{4}+\frac{1}{2}\left(c_{2}-\frac{c_{1}^{2}}{2}\right) Q_{1}\right\} z^{2}+\cdots .
$$


Using (3.14) in (3.13) and along with (1.3), we obtain

$$
a_{p+1}=\frac{p Q_{1} c_{1}}{[2 p]_{q}^{m}\left\{[p+1]_{q}-p\right\}}
$$

and

$$
a_{p+2}=\frac{p}{[2 p+1]_{q}^{m}\left\{[p+2]_{q}-p\right\}}\left\{\frac{Q_{1} c_{2}}{2}+\frac{c_{1}^{2}}{4}\left(Q_{2}-Q_{1}+\frac{4 p Q_{1}^{2}}{\left\{[p+1]_{q}-p\right\}}\right)\right\} .
$$

Using any complex number $\mu$ and the above coefficients, we have

$$
a_{p+2}-\mu a_{p+1}^{2}=\frac{p Q_{1}}{2[2 p+1]_{q}^{m}\left\{[p+2]_{q}-p\right\}}\left\{c_{2}-v c_{1}^{2}\right\} .
$$

Using Lemma 2.3 on (3.15), we have

$$
\left|a_{p+2}-\mu a_{p+1}^{2}\right| \leq \frac{p Q_{1}}{2[2 p+1]_{q}^{m}\left\{[p+2]_{q}-p\right\}} \max [1,|2 v-1|],
$$

where

$$
v=\frac{1}{2}\left\{1-\frac{Q_{2}}{Q_{1}}-Q_{1}\left(\frac{4 p}{\left\{[p+1]_{q}-p\right\}}-\mu \frac{4 p[2 p+1]_{q}^{m}\left\{[p+2]_{q}-p\right\}}{\left([2 p]_{q}^{m}\right)^{2}\left\{[p+1]_{q}-p\right\}}\right)\right\} .
$$

This is our required result (3.11).

When $p=1$, we have the following known result proved by Saqib et al. in [12].

Corollary 3.6 ([12]) Let $0 \leq k<\infty$ be fixed and let $f(z) \in k-\mathcal{U S}(q, \gamma, m)$ with the form (1.1). Then, for a complex number $\mu$,

$$
\left|a_{3}-\mu a_{2}^{2}\right| \leq \frac{Q_{1}}{2[3]_{q}^{m}\left\{[3]_{q}-1\right\}} \max [1,|2 v-1|]
$$

where

$$
v=\frac{1}{2}\left\{1-\frac{Q_{2}}{Q_{1}}-Q_{1}\left(\frac{4}{[2]_{q}-1}-\mu \frac{4[3]_{q}^{m}\left\{[3]_{q}-1\right\}}{\left([2]_{q}^{m}\right)^{2}\left\{[2]_{q}-1\right\}}\right)\right\},
$$

and $Q_{1}$ and $Q_{2}$ are given by (2.2) and (2.3).

Theorem 3.7 If a function $f(z) \in \mathcal{A}_{p}$ has the form (1.1) and satisfies the condition

$$
\sum_{n=p+1}^{\infty}\left\{\left\{\left|[n]_{q}-p\right|\right\}(k+1)+p|\gamma|\right\}\left|\psi_{n}\right|\left|a_{n}\right| \leq|\gamma||p|
$$

then $f(z) \in k-\mathcal{U S}(q, \gamma, m, p)$. 
Proof Let

$$
\begin{aligned}
\left|\frac{1}{p}\left(\frac{z \partial_{q} S_{q, p}^{m} f(z)}{S_{q, p}^{m} f(z)}\right)-1\right| & =\left|\frac{z \partial_{q} S_{q, p}^{m} f(z)-p S_{q, p}^{m} f(z)}{p S_{q, p}^{m} f(z)}\right| \\
& =\left|\frac{\sum_{n=p+1}^{\infty} \psi_{n}\left\{[n]_{q}-p\right\} a_{n} z^{n}}{p z^{p}+p \sum_{n=p+1}^{\infty} \psi_{n} a_{n} z^{n}}\right| \\
& \leq \frac{\sum_{n=p+1}^{\infty}\left|\psi_{n}\left\{[n]_{q}-p\right\}\right|\left|a_{n}\right|}{|p|-\sum_{n=p+1}^{\infty} p\left|\psi_{n}\right|\left|a_{n}\right|} .
\end{aligned}
$$

From (3.16), it follows that

$$
p-\sum_{n=p+1}^{\infty} p\left|\psi_{n}\right|\left|a_{n}\right|>0 .
$$

To show that $f(z) \in k-\mathcal{U S}(q, \gamma, m, p)$, it is enough to prove that

$$
\left|\frac{k}{\gamma}\left\{\frac{1}{p}\left(\frac{z \partial_{q} S_{q, p}^{m} f(z)}{S_{q, p}^{m} f(z)}\right)-1\right\}\right|-\operatorname{Re}\left\{\frac{1}{\gamma}\left\{\frac{1}{p}\left(\frac{z \partial_{q} S_{q, p}^{m} f(z)}{S_{q, p}^{m} f(z)}\right)-1\right\}\right\} \leq 1 .
$$

From (3.17), we have

$$
\begin{aligned}
\mid \frac{k}{\gamma} & \left\{\frac{1}{p}\left(\frac{z \partial_{q} S_{q, p}^{m} f(z)}{S_{q, p}^{m} f(z)}\right)-1\right\} \mid-\operatorname{Re}\left\{\frac{1}{\gamma}\left\{\frac{1}{p}\left(\frac{z \partial_{q} S_{q, p}^{m} f(z)}{S_{q, p}^{m} f(z)}\right)-1\right\}\right\} \\
& \leq \frac{k}{|\gamma|}\left|\frac{1}{p}\left(\frac{z \partial_{q} S_{q, p}^{m} f(z)}{S_{q, p}^{m} f(z)}\right)-1\right|+\frac{1}{|\gamma|}\left|\frac{1}{p}\left(\frac{z \partial_{q} S_{q, p}^{m} f(z)}{S_{q, p}^{m} f(z)}\right)-1\right| \\
& \leq \frac{(k+1)}{|\gamma|}\left|\frac{1}{p}\left(\frac{z \partial_{q} S_{q, p}^{m} f(z)}{S_{q, p}^{m} f(z)}\right)-1\right| \\
& =\frac{(k+1)}{|\gamma|}\left|\frac{z \partial_{q} S_{q, p}^{m} f(z)-p S_{q, p}^{m} f(z)}{p S_{q, p}^{m} f(z)}\right| \\
& \leq \frac{(k+1)}{|\gamma|}\left\{\frac{\sum_{n=p+1}^{\infty}\left|\psi_{n}\left\{[n]_{q}-p\right\}\right|\left|a_{n}\right|}{|p|-\sum_{n=p+1}^{\infty} p\left|\psi_{n}\right|\left|a_{n}\right|}\right\} \\
& \leq 1 .
\end{aligned}
$$

When $p=1$, we have the following known result proved by Hussain et al. in [12].

Corollary 3.8 ([12]) If a function $f(z) \in \mathcal{A}$ has the form (1.1) and satisfies the condition

$$
\sum_{n=2}^{\infty}\left\{\left|[n]_{q}-1\right|(k+1)+|\gamma|\right\}[n]_{q}^{m}\left|a_{n}\right| \leq|\gamma|
$$

then $f(z) \in k-\mathcal{U S}(q, \gamma, m)$.

When $q \rightarrow 1, p=1, m=0, \gamma=1-\alpha$, with $0 \leq \alpha<1$, we have the following known result, proved by Shams et al. in [28]. 
Corollary 3.9 A function $f \in A$ of the form (1.1) is in the class $\mathcal{S D}(k, \alpha)$ if it satisfies the condition

$$
\sum_{n=2}^{\infty}\{n(k+1)-(k+\alpha)\}\left|a_{n}\right| \leq 1-\alpha,
$$

where $0 \leq \alpha<1$ and $k \geq 0$.

When $q \rightarrow 1, p=1, m=0, \gamma=1-\alpha$, with $0 \leq \alpha<1$ and $k=0$, we have the following known result proved by Silverman in [30].

Corollary 3.10 A function $f \in A$ of the form (1.1) is in the class $\mathcal{S D}(\alpha)$ if it satisfies the condition

$$
\sum_{n=2}^{\infty}\{n-\alpha\}\left|a_{n}\right| \leq 1-\alpha .
$$

Theorem 3.11 Let $f(z) \in k-\mathcal{U S}(q, \gamma, m, p)$. Then $f(E)$ contains an open disk of radius

$$
r=\frac{\left\{[p+1]_{q}-p\right\} \psi_{p+1}}{(p+1)\left\{[p+1]_{q}-p\right\} \psi_{p+1}+\delta},
$$

where $\delta=p\left|Q_{1}\right|$ with $Q_{1}$ given by (2.2).

Proof Let $w_{0} \neq 0$ be a complex number such that $f(z) \neq w_{0}$ for $z \in E$. Then

$$
f_{1}(z)=\frac{w_{0} f(z)}{w_{0}-f(z)}=z+\left(a_{p+1}+\frac{1}{w_{0}}\right) z^{p+1}+\cdots .
$$

Since $f_{1}(z)$ is univalent, so

$$
\left|a_{p+1}+\frac{1}{w_{0}}\right| \leq p+1
$$

Now, by using (3.6), we have

$$
\left|\frac{1}{w_{0}}\right| \leq \frac{(p+1)\left\{[p+1]_{q}-p\right\} \psi_{p+1}+\delta}{\left\{[p+1]_{q}-p\right\} \psi_{p+1}} .
$$

Hence we have

$$
\left|w_{0}\right| \geq \frac{\left\{[p+1]_{q}-p\right\} \psi_{p+1}}{(p+1)\left\{[p+1]_{q}-p\right\} \psi_{p+1}+\delta} .
$$

When $p=1$, we have the following known result proved by Saqib et al. in [12].

Corollary 3.12 ([12]) Let $f(z) \in k-\mathcal{U S}(q, \gamma, m)$. Then $f(E)$ contains an open disk of radius

$$
r=\frac{\left\{[2]_{q}-1\right\}[2]_{q}^{m}}{2[2]_{q}^{m}\left\{[2]_{q}-1\right\}+Q_{1}},
$$

where $Q_{1}$ is given by (2.2). 
Funding

The work here is supported by GUP-2017-064 and UKM Grant Nos. DPP-2015-FST.

Competing interests

The authors declare that there are no competing interests.

\section{Authors' contributions}

SH came with the main thoughts and helped to draft the manuscript, SK and MAZ proved the main theorems, MD revised the paper. All authors read and approved the final manuscript.

\section{Author details}

'Department of Mathematics, COMSATS University Islamabad, Abbottabad Campus, Pakistan. ${ }^{2}$ Department of Mathematics, Riphah International University, Islamabad, Pakistan. ${ }^{3}$ Faculty of Science and Technology, School of Mathematical Sciences, Universiti Kebangsaan Malaysia, Bangi, Malaysia.

\section{Publisher's Note}

Springer Nature remains neutral with regard to jurisdictional claims in published maps and institutional affiliations.

Received: 17 July 2018 Accepted: 23 October 2018 Published online: 07 November 2018

\section{References}

1. Adams, C.R.: On the linear partial q-difference equation of general type. Trans. Am. Math. Soc. 31, 360-371 (1929)

2. Agrawal, S., Sahoo, S.K.: A generalization of starlike functions of order $\alpha$. Hokkaido Math. J. 46, 15-27 (2017)

3. Ali, R.M.: Starlikeness associated with parabolic regions. Int. J. Math. Math. Sci. 4, 561-570 (2005)

4. Anastassiou, G.A., Gal, S.G.: Geometric and approximation properties of generalized singular integrals in the unit disk. J. Korean Math. Soc. 43(2), 425-443 (2006)

5. Andrews, G.E., Askey, G.E., Roy, R.: Special Functions. Cambridge University Press, Cambridge (1999)

6. Aral, A., Gupta, V.: On the generalized Picard and Gauss Weierstrass singular integrals. J. Comput. Anal. Appl. 8(3), 249-261 (2006)

7. Aral, A., Gupta, V.: On q-Baskakov type operators. Demonstr. Math. 42(1), 109-122 (2009)

8. Aral, A., Gupta, V.: On the Durrmeyer type modification of $q$-Baskakov type operators. Nonlinear Anal., Theory Methods Appl. 73(3), 1171-1180 (2010)

9. Aral, A., Gupta, V.: Generalized q-Baskakov operators. Math. Slovaca 61(4), 619-634 (2011)

10. Carmichael, R.D.: The general theory of linear q-difference equations. Am. J. Math. 34, 147-168 (1912)

11. Govindaraj, M., Sivasubramanian, S.: On a class of analytic functions related to conic domains involving $q$-calculus. Anal. Math. https://doi.org/10.1007/s10476-017-0206-5

12. Hussain, S., Khan, S., Zaighum, M.A., Darus, M.: Certain subclass of analytic functions related with conic domains and associated with Salagean q-differential operator. AIMS Math. 2(4), 622-634 (2017)

13. Jackson, F.H.: On $q$-functions and certain differences operator. Earth Environ. Sci. Trans. R. Soc. Edinb. 46(2), 253-281 (1909)

14. Jackson, F.H.: On q-definite integrals. Pure Appl. Math. Q. 41(15), 193-203 (1910)

15. Kanas, S., Raducanu, D.: Some class of analytic functions related to conic domains. Math. Slovaca 64(5), 1183-1196 (2014)

16. Kanas, S., Wisniowska, A.: Conic regions and k-uniform convexity. J. Comput. Appl. Math. 105, 327-336 (1999)

17. Kanas, S., Wisniowska, A.: Conic domains and k-starlike functions. Rev. Roum. Math. Pures Appl. 45, 647-657 (2000)

18. Ma, W., Minda, D.: A unified treatment of some special classes of univalent functions. In: Li, Z., Ren, F., Yang, L., Zhang, S. (eds.) Proceedings of the Conference on Complex Analysis, pp. 157-169. International Press, Somerville (1992)

19. Mahmood, S., Darus, M.: Some subordination results on $q$-analogue of Ruscheweyh differential operator. Abstr. Appl. Anal. 2014, Article ID 958563 (2014)

20. Mahmood, S., Sokol, J.: New subclass of analytic functions in conical domain associated with Ruscheweyh q-differential operator. Results Math. (2016)

21. Masjed-Jamei, M., Koepf, W.: Some summation theorems for generalized hypergeometric functions. Axioms 7, 38 (2018)

22. Mason, T.E.: On properties of the solution of linear $q$-difference equations with entire function coefficients. Am. J. Math. 37, 439-444 (1915)

23. Noor, K.I., Arif, M., Ul-Haq, W.: On k-uniformly close-to-convex functions of complex order. Appl. Math. Comput. 215, 629-635 (2009)

24. Owa, S., Polatoglu, Y., Yavuz, E.: Coefficient inequalities for classes of uniformly starlike and convex functions. J. Inequal. Pure Appl. Math. 7(5), 1-5 (2006)

25. Rogosinski, W.: On the coefficients of subordinate functions. Proc. Lond. Math. Soc. 48, 48-82 (1943)

26. Salagean, G.S.: Subclasses of univalent functions. In: Complex Analysis, Fifth Romanian-Finnish Seminar, Part 1 , Bucharest, 1981. Lecture Notes in Mathematics, vol. 1013, pp. 362-372. Springer, Berlin (1983)

27. Seoudy, T.M., Aouf, M.K.: Coefficient estimates of new classes of $q$-starlike and $q$-convex functions of complex order. J. Math. Inequal. 10(1), 135-145 (2016)

28. Shams, S., Kulkarni, S.R., Jahangiri, J.M.: Classes of uniformly starlike and convex functions. Int. J. Math. Math. Sci. 55, 2959-2961 (2004)

29. Shareef, Z., Hussain, S., Darus, D.: Convolution operator in geometric functions theory. J. Inequal. Appl. 2012, 213 (2012)

30. Silverman, H.: Univalent functions with negative coefficients. Proc. Am. Math. Soc. 51, 109-116 (1975)

31. Sim, S.J., Kwon, O.S., Cho, N.E., Srivastava, H.M.: Some classes of analytic functions associated with conic regions. Taiwan. J. Math. 16(1), 387-408 (2012)

32. Trjitzinsky, W.J.: Analytic theory of linear q-difference equations. Acta Math. 61, 1-38 (1933) 